\title{
Joint Mission Space Entity of Military Analysis Simulation and Evaluation System
}

\author{
Xinye Zhao ${ }^{1, a}$, Haiming Liang ${ }^{1, b}$, Dong Wang ${ }^{2, c}$, Tianjia Liü, \\ ${ }^{1}$ Dalian Naval Academy, Dalian 116018, China \\ ${ }^{2}$ Naval Aeronautical and Astronautical University, Yantai 264001, China \\ ${ }^{3}$ PLA Army 91550, Dalian 116018, China \\ azhaoxinye@nudt.edu.cn, ${ }^{\mathrm{b}}$ lianghaiming_1987@sina.com, ${ }^{\mathrm{c}} \mathrm{wd} 124$ nudt@sina.com, \\ dnomad_angel@126.com
}

Keywords: Military Analysis Simulation and Evaluation System; Knowledge Base; Command \& Control; Joint Mission Space Entity.

\section{Abstract.}

Military simulation analysis and evaluation system (MASES) may integrate simulation into real Command \& Control system. JMSEs could depict various entities of the real world and carry out their full functional modeling, which can be regarded as the function unit of MASES. This paper describes the concept of JMSE, JMSE class, JMSE attribute, JMSE asset, JMSE type, etc., and how our approach reduces the time and effort required to compose simulations. Recent simulation systems have underscored the need for that command and control model are based on "perceived facts", not necessarily "real facts" or "objective facts", while JMSE could offer that capability. Furthermore, for the purpose of proving the validity of the proposed method, an application of Maritime JMSE is presented for a unit damage algorithm. This is a major step forward in the command and control of the previous campaign-level simulation based on the "real facts".

\section{Introduction}

Several attempts have been made in the past decades to provide a higher level operational simulation of large-scale joint operations and diversification of military mission planning implementation. Thus, military analysis simulation evaluation system (MASES) [1] should have multiple combat power, multi-dimensional battlefield space, a variety of action patterns, all kinds of security system integration based on information system simulation evaluation ability. MASES uses three software domains to model the joint task space: Problem, Platform, and Simulation. The three software domains are integrated into a complete execution software Package to complete the battle research and analysis [2,3]. The problem domain is the focus of this volume, which constructs the entities and interactions of the combat area. The second volume deals with the platform (HCI), the simulation domain provides the system architecture and common code. As an integral part of the MASES problem domain, JMSEs (Joint Mission Space Entity) are an indispensable part of the MASES model. JMSEs depict real-world entities and have fully functional modeling of them, and thus can be considered as functional actors in MASES.

\section{Brief of MASES}

In the new era of information, the military analysis simulation evaluation system needs to complete the mission, including for the coarse-grained model combat program derivation optimization, such as exploratory analysis of simulation; for combat planning inspection, statistical evaluation and analysis; online support decision support; military skill building. Due to the particularity of the application of military analysis and simulation system, the functional requirements of modeling and simulation must be domain object-oriented modeling, which ensures the plug and play of simulation components. It is in the conjugation of these efforts that some of the most interesting questions arise. From the original static configuration can be static configuration can 
also be dynamic group. The dynamic structure reconfiguration is an important feature of complex system simulation.

Due to the three-level classification of MASES, where the problem domain provides software for analysis purposes that can describe combat functions. The simulation domain provides the "engine" that drives the simulation run, but also provides a three-dimensional battlefield space description. The battlefield space is the "Synthetic Natural Environment (SNE)" where JMSEs live. The platform domain provides MASES hardware and helps the person or other person acquire the Human Computer Interface (HCI) for emulated input/output data,

\section{JMSE}

\section{Definition of JMSE}

JMSEs depict real-world entities and have fully functional modeling of them, and thus can be considered as functional actors in MASES. In MASES system, the military component and the system's constituent modules are called Joint Mission Space Entity (JMSE). The resolution of the JMSE's land-based exercise unit is camp, air combat is flight formation, sea power is ship, and key intelligence reconnaissance surveillance (ISR) system is a single platform. In addition, there are some special JMSE, such as ports, airports, major commanders (such as division headquarters) and chemical clouds. JMSE data expresses the static and dynamic properties of an entity. Static data expresses values that do not change over time, such as the legal strength of the combat unit, the firepower range of the missile system. Dynamic data (such as force combat, location, etc.) can change over time. These data also indicate the interaction between JMSE and each other and its interaction with the environment.

An excerpt of JMSE model composition is shown in Fig. 1. JMSE model consists of Unit, Asset, Installation and other components. Messages are transferred by $\mathrm{C} 2$ (Command \& Control) [4,5] relationships between different kinds of components. By composing different assets component, MASES could construct different functional models flexibly.

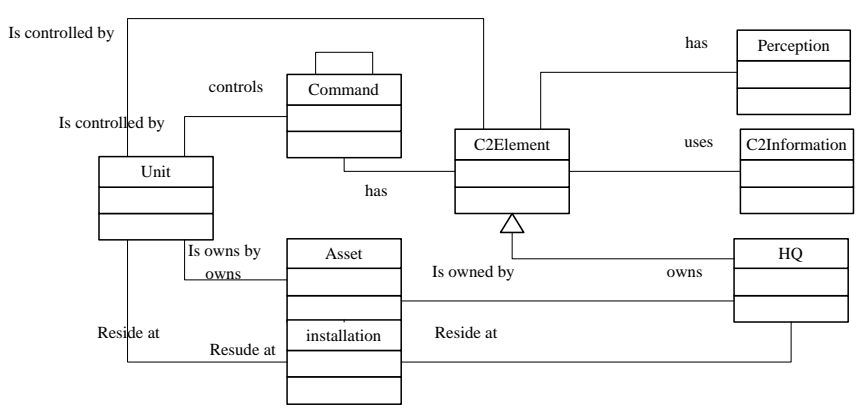

Fig.1 JMSE model composition

All JMSE entities have command and control capabilities. Please refer to Fig.2 for a better visualization of JMSE definition structure. Other objects in the simulation that describe entities in the real world can only model the "slaved" assets of the JMSE (i.e. only the details of a hierarchy that conforms to the JMSE assets). Such as Fire Concentration Points; other examples include tanks and transport vehicles, etc., which is set as assets of the JMSE (i.e. attribute data for each JMSE object), rather than as the object itself.

\section{JMSE Classes}

C2 - JMSE class, which contains the command and control (C2) behavior of the JMSE. The two terms related to object-oriented (OO) programming are: subclasses and super-classes. Subclasses are passed directly or indirectly from a given class, or special class from the base class; super-class is 
directly or indirectly through the inheritance and get the class given by the class from the point of view, JMSE is the highest level of "C2", which provides the framework and functionality of all the simulation entities, the JMSE class from their superclass inherits the public function (behavior), in general, each JMSE class gradually adds more (or modifies the default) of the function defined by its super-class, which, like the object-oriented (OO) software design, can reuse objects and describe new objects without having to constantly rebuild or define them.

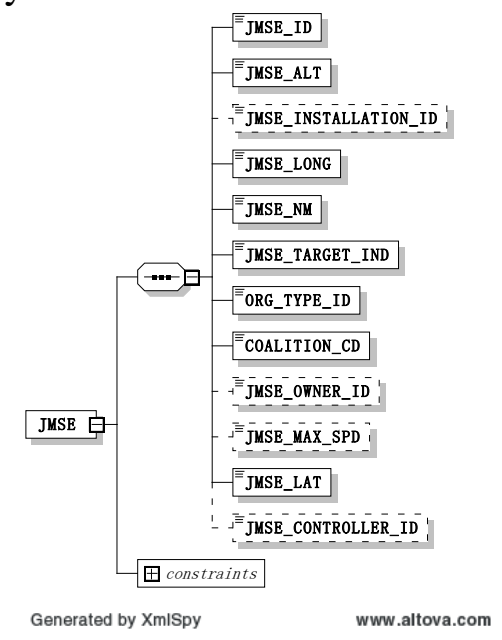

Fig.2 JMSE Definition Structure

\section{JMSE Attributes}

Attributes are characteristics that describe the physical composition of things (eg size, equipment, assets), inherited features (eg, unit functions, their countries), or behavior (eg speed, formation).

The key attributes inherited by the JMSE from the JMSE type include:

- OrgType ID: unique identifier for the organization (type) type (also called JMSE type)

- Assets: means resources, inherited assets list, is referred to as from OrgTypeAsset;

- C2: means behavior, description of the package has a simulation of the entity command control behavior of the class;

- Category: description of the higher class (such as: ground, air, sea, etc);

- Level: echelons of land formation template, first applied to land (from connecting to division);

- Radius: the distance to the JMSE center (longitude/latitude), which indicates the size of the JMSE, the default unit is meters, the land combat JMSE first derives the radius from the formation template;

- Icon: Describe the symbol of the operation;

- Sensor: sensor type (name), can detect, judge and identify other JMSE.

\section{JMSE Assets}

Assets are controlled, possessed, reserved by JMSE, so that JMSE can express specific and behavior or according to the algorithm was ruled. Fig.2 lists the structure of MASES assets. MASES assets are complex simulation of different objects, each Assets have one to more than the following components:

- Asset Specification: Description of a specific asset data;

- Asset Cargo Characteristics: the asset characteristics when the assets are packaged or disposed on a transport carrier;

- Asset Ballistic Missile: tactical ballistic missile data;

- Asset Naval Weapon (resources naval weapons): ship gun and missile data;

- Asset Towed Array (resource drag display): the tail of the towing sensor display data;

- Asset Platform (resource platform): designed as the platform resource characteristics. 


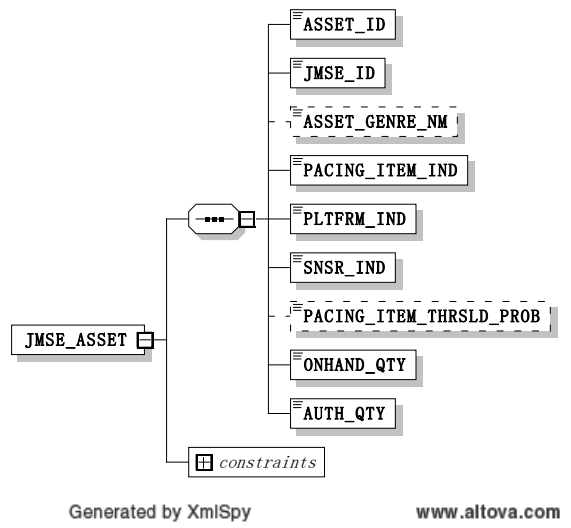

Fig.2 JMSE Asset Structure

\section{JMSE Types}

JMSEs inherit attributes from their organization type (OrgType or JMSEType). OrgType (or JMSEType) is the general type or class of force units, JMSEType provides templates for individual JMSE instances, common OrgType concepts such as organization and equipment forms, Standard Requirement Code, Unit Type Code. The JMSE instance corresponds to a modified organization and equipment forms.

\section{Application of Maritime JMSE}

Maritime JMSE's C2 model has the characteristics of the captain's online command and the composite combat command, which receives the maritime combat mission command sent by the Joint Forces C2 model, distributes the ship, sets up a task-specific special formation, establish the maritime operations plan, and assigned to the lower commanders specific tasks. If necessary, maritime JMSE's C2 model can coordinate the subordinate combat commander to complete the combat mission; in response to the command and control model from the joint force command and control, the joint force army command and control model, the joint force air force command and control model command or request; in the joint force command and control model, unify other arms of services to complete the joint combat mission. To create a maritime task force for the way, the organization of maritime combat forces, the formation of maritime operations command level relationship. In fact, it is from the joint mission organization JFMCC (Joint Forces Maritime Division Commander) part of the separate operation. The membership of JMSE could be changed as well, to achieve the task group membership adjustment.

Taking the damage mechanism of sea unit as an example, this paper introduces the creation and use of Maritime JMSE. Components that affect Maritime JMSE performance and behavior include: Maritime C2 and Weapon System。Maritime surface warfare requires characteristics as follows:

C2Architecture concludes at least one MaritimeTaskOrientedGroupJMSE, and MaritimeTaskOrientedGroupJMSE includes one SurfaceWarefareCommanderJMSE, while SurfaceWarefareCommanderJMSE includes one or more Maritime Combat Vessel. Maritime undersea warfare requires characteristics as follows: C2Architecture concludes at least one MaritimeTaskOrientedGroupJMSE, and MaritimeTaskOrientedGroupJMSE includes one UnderseaWarefareCommanderJMSE, while UnderseaWarefareCommanderJMSE includes one or more Maritime Combat Vessel. Every combat vessel must concludes proper weapons and sensors for surface or undersea warfare, while surface warfare only needs AswCommandCenter C2 plugin to accmplish USW.

Once the enemy and naval targets are acquired, maritime JMSE will use weapons to engage with it. The naval-capable naval JMSEs not only include combatants, but also auxiliary ships and motor ships. The type of weapon used depends on the attack and target JMSE type. The matching scheme of Attack JMSEType and Target JMSEType is showed as Tab.1. 
Table 1 matching scheme of Attack JMSEType and Target JMSEType

\begin{tabular}{|c|c|c|}
\hline Attack JMSEType & \multicolumn{2}{|c|}{ Target JMSEType } \\
\hline Surface & Surface & Submarine \\
\hline Surface-to-Surface Missile & Taval guns & \\
\hline Submarine & Torpedo & \\
\hline
\end{tabular}

Due to a lack of space, some technical details have been left out of this paper. The fire algorithm for ship and submarine attacks, and the random algorithm determines whether to kill is restricted as follows:

- For ship-to-ship missile and fire attack decisions, the algorithm uses a joint ammunition effect manual and other data to perform a random assessment. Probability table gives the result by attacking the number of missiles. Disastrous killings and other damage states are calculated from random sampling.

- For the submarine attack on submarine missiles, the use of the Maritime to provide the probability of killing the table, randomly selected decision results.

- The mine decision uses a traditional negative exponential probability to determine whether the mine is detonated, according to the mine's density and depth. The probability of probabilities is used to determine whether the mine is detonated. Determine the detonation, random extraction is used to determine the actual effect.

\section{Conclusion}

This paper addresses a complex problem with requirements spanning diverse areas of knowledge about operational simulation. In MASES system, the interaction between all JMSEs is scheduled as a simulation event. The importance of a single event can be from "not very important" to "very important". Information (multiple data) fusion problem using Bayes technology to establish probability model processing. And those events (which have an important effect on battle-level decision-making on the right side of the particle resolution spectrum) are also evaluated using discrete algorithms using discrete output results. The next study will focus on multi-JMSE operation of the data fusion problem, in order to better support MASES support decision-making function.

\section{Acknowledgements}

This work is supported by China Postdoctoral Science Foundation under Grant No.2016M602962 and No. 2017 T100797.

\section{References}

[1] Kedi Huang, Xinye Zhao, Shanliang Yang, et al. System Design Description Infrastructure Overview for Military Simulation and Analysis System[J] Journal of System Simulation. 2012, 24(12): 2439-2447.

[2] Blacksten H R, Jones J W, Poumade M L, et al. JWARS Output Analysis[C]. Proceedings of the Winter Simulation Conference. IEEE, 2001(1): 706-714.

[3] Metz M L. Joint Warfare System (JWARS) Verification And Validation Lessons Learned[C]. Proceedings of the Winter Simulation Conference. IEEE, 2001(1): 855-858.

[4] Madjid T, Trevisani D A, Kennedy D T. A Fuzzy Cyber-Risk Analysis Model for Assessing Attacks on the Availability and Integrity of the Military Command and Control Systems[J] Japanese Journal Of Human Sciences Of Health-Social Services, 2015, 1(3):21-36.

[5] Mcdermid J A. Distributed Databases and Command and Control Systems[J]. Hydrology \& Earth System Sciences, 2016, 2(4):509-520. 